\title{
Prevalence of serrated polyposis syndrome and its association with synchronous advanced adenoma and lifestyle
}

\author{
NAOYA TOYOSHIMA ${ }^{1,2}$, TAKU SAKAMOTO ${ }^{1}$, MAKOMO MAKAZU $^{1}$, TAKESHI NAKAJIMA $^{1}$, \\ TAKAHISA MATSUDA ${ }^{1}$, RYOJI KUSHIMA ${ }^{3}$, TADAKAZU SHIMODA ${ }^{4}$, TAKAHIRO FUJII ${ }^{5}$, \\ HARUHIRO INOUE $^{2}$, SHIN-EI KUDO ${ }^{2}$ and YUTAKA SAITO ${ }^{1}$ \\ ${ }^{1}$ Endoscopy Division, National Cancer Center Hospital, Tokyo 104-0045; ${ }^{2}$ Digestive Disease Center, \\ Showa University Northern Yokohama Hospital, Yokohama, Kanagawa 224-8503; ${ }^{3}$ Division of Molecular Pathology, \\ National Cancer Center Research Institute, Tokyo 104-0045; ${ }^{4}$ Division of Molecular Pathology, National Cancer Center \\ Research Institute, Tokyo 104-0061; ${ }^{5}$ TF Clinic, Tokyo 104-0061, Japan
}

Received July 18, 2014; Accepted September 12, 2014

DOI: $10.3892 / \mathrm{mco} .2014 .423$

\begin{abstract}
We assessed the clinicopathological characteristics of patients with serrated polyposis syndrome (SPS) and the incidence of advanced adenoma/colorectal cancer (CRC). We prospectively enrolled 249 consecutive patients who underwent colonoscopy at the National Cancer Center Hospital over a 6-month period. All the polyps were diagnosed using magnification colonoscopy and resection/biopsy. The enrolled patients were divided into two groups, i) those with $\geq 5$ histologically diagnosed hyperplastic polyps (HPs) proximal to the sigmoid colon, with at least 2 polyps $>10 \mathrm{~mm}$ in diameter and ii) those with $\geq 20$ HPs distributed throughout the colon. The clinical characteristics of the two groups were compared, including lifestyle, family history of CRC and colonoscopic findings. HPs were identified in 228 patients, of whom $21(8.4 \%)$ had SPS. All 21 patients had $\geq 20$ HPs distributed throughout the colon, with none having $>2 \mathrm{HPs} \geq 1 \mathrm{~cm}$ in diameter in the right colon. Synchronous advanced adenoma/CRC was diagnosed in 76/249 (30.5\%) patients. The prevalence of advanced adenoma/CRC was higher among patients with compared to those without SPS ( $\mathrm{P}=0.075)$. SPS was also associated with older age and higher body mass index (BMI). Our results suggested that older age and higher BMI are independent risk factors for SPS. Advanced adenoma/CRC tended to occur more frequently among patients with compared to those without SPS, although the difference was not statistically significant.
\end{abstract}

Correspondence to: Dr Yutaka Saito, Endoscopy Division, National Cancer Center Hospital, 5-1-1 Tsukizi, Chuo-ku, Tokyo 104-0045, Japan

E-mail: ytsaito@ncc.go.jp

Key words: colonoscopy, colorectal cancer, pit pattern, serrated polyposis syndrome

\section{Introduction}

Hyperplastic polyps (HPs) were considered as harmless, with no potential of progressing to colorectal cancer (CRC) (1). Recently, however, serrated polyps (i.e., HPs, sessile serrated adenomas and traditional serrated adenomas) were reported to progress to CRC via the serrated neoplasia pathway (2-4). Large HPs in the right colon have been associated with synchronous CRC and have exhibited malignant potential (5-9).

Hyperplastic polyposis syndrome (HPS) is a clinical condition characterized by the presence of multiple HPs throughout the colorectum and has been associated with increased risk of CRC. The diagnostic criteria of HPS were first described by Burt and Jass (5) in 2000 for the World Health Organization (WHO). These criteria were recently redefined and this condition is currently referred to as serrated polyposis syndrome (SPS). A number of patients have been reported as having CRC and concurrent SPS (10-16), suggesting that SPS may be an alternative pathway for the development of CRC. However, SPS has not yet been extensively investigated in Japan and its clinical characteristics, causes and association of SPS to CRC have not been adequately evaluated.

The prevalence of SPS has been reported to range between 0.01 and $0.05 \%$, but has not yet been assessed by magnification chromoendoscopy. Accurate detection of HPs throughout the colorectum depends on the quality of the colonoscopic examination, which may be limited by visibility, bowel preparation, spraying with indigo carmine dye and magnification (9).

We previously investigated the prevalence of SPS by using magnification chromoendoscopy. In this study, we aimed to evaluate SPS based on WHO clinical criteria and assess its prevalence, clinicopathological characteristics and association with advanced adenoma/CRC.

\section{Material and methods}

Study population. We prospectively enrolled 249 consecutive patients who underwent initial total colonoscopy at the National Cancer Center Hospital, Tokyo, Japan, over a 6-month period. The indications for colonoscopy are listed 
in Table I. Patients were excluded if they had been previously treated for a colorectal neoplasm at another hospital; if they had a personal or family history of familial adenomatous polyposis, or any hereditary disease; if they had previously undergone a colectomy; or if they regularly used non-steroidal anti-inflammatory drugs.

Informed consent was obtained from all the patients and our Institutional Review Board and Institutional Medical Ethics Committees approved the study protocol, which adhered to the tenets of the Declaration of Helsinki.

Methods. All the patients underwent initial total colonoscopy using a magnifying video colonoscope (PCF-Q240Z; Olympus Optical Co., Tokyo, Japan). Spray catheters were used to spray indigo carmine dye from the cecum to the rectum in all the patients. The location, size and number of HPs and neoplastic lesions were recorded. All the polyps were diagnosed using magnifying colonoscopy and resected or biopsied for histopathological analysis. The enrolled patients were divided into two groups based on SPS criteria. Patients were diagnosed with SPS if they had $\geq 20$ HPs distributed throughout the colon and/or $\geq 5$ histologically diagnosed HPs proximal to the sigmoid colon, with at least 2 polyps $>10 \mathrm{~mm}$ in diameter. Each patient completed a structured questionnaire, which included information on lifestyle and familial history of CRC. Lifestyle questions included age, gender, body mass index (BMI) and the amount and duration of cigarette smoking and alcohol consumption during the patient's lifetime. Smokers were defined as those having ever smoked and drinkers were defined those who drank $\geq 30 \mathrm{ml}$ alcohol per day for $\geq 5$ years (the following assumptions and conversion rates were used: $1 \mathrm{oz}$ was equivalent to $30 \mathrm{ml} ; 1$ glass of wine contains $120 \mathrm{ml}$; 1 bottle of beer contains $360 \mathrm{ml}$; and 1 drink of hard liquor contains $45 \mathrm{ml}$; the amount of absolute alcohol consumed per day was calculated using conversion rates of $10 \%$ for wine, $3.7 \%$ for beer and $38 \%$ for hard liquor).

Statistical analyses. Our primary aim was to estimate the relative risk, using odds ratio (OR) as an approximation, of SPS associated with lifestyle. We calculated the ORs and $95 \%$ confidence intervals associated with age, gender, BMI, smoking, alcohol consumption and family history of CRC using unconditional multivariate logistic regression analysis. $\mathrm{P}<0.05$ was considered to indicate a statistically significant difference and all the tests for statistical significance were two-sided. All the statistical analyses were performed using Stata software, version 10 (StataCorp, College Station, TX, USA).

\section{Results}

Patients and lifestyle. Of the 249 patients (130 men and 119 women), 228 (91.6\%) had HPs; of the latter, 21 (8.4\%) had SPS, all of whom had $\geq 20$ HPs distributed throughout the colon and none having $>2$ HPs $\geq 1 \mathrm{~cm}$ in diameter in the right colon.

The clinical characteristics of the patients are summarized in Table II. The SPS group consisted of 15 men and 6 women (median age, 66 years; median BMI, $23.7 \mathrm{~kg} / \mathrm{m}^{2}$ ). The proportion of current alcohol consumers in the SPS and non-SPS groups was $0 \%(0 / 21)$ and $11.5 \%(25 / 228)$, respectively. There
Table I. Indications for colonoscopy.

Indications

No. of patients

Screening

42

Positive fecal occult blood test or melena

76

Abdominal symptoms

41

Further examination of the colorectal lesion

84

Other

6

Total

249

Table II. Clinical characteristics of patients with and without SPS.

\begin{tabular}{|c|c|c|c|}
\hline Characteristics & $\begin{array}{c}\text { SPS } \\
(n=21)\end{array}$ & $\begin{array}{l}\text { Non-SPS } \\
(n=228)\end{array}$ & P-value \\
\hline BMI & & & $<0.01$ \\
\hline Median & 23.7 & 22.2 & \\
\hline Range & $23.1-25.5$ & $20.0-24.2$ & \\
\hline Age, years & & & 0.018 \\
\hline Median (range) & 66 & 60 & \\
\hline Range & $59-70$ & $51-67$ & \\
\hline Gender, no. (\%) & & & 0.065 \\
\hline Male & $15(71.4)$ & $115(50.4)$ & \\
\hline Female & $6(28.6)$ & 113 (49.6) & \\
\hline ADA Ca, no. (\%) & & & 0.075 \\
\hline Present & $10(47.6)$ & $66(28.9)$ & \\
\hline Absent & $11(52.4)$ & $162(71.1)$ & \\
\hline Smoking, no. (\%) & & & 0.241 \\
\hline Never & $10(47.6)$ & $148(62.3)$ & \\
\hline Current or ever & $11(52.4)$ & $80(37.7)$ & \\
\hline Alcohol, no. (\%) & & & 0.101 \\
\hline No & $21(100)$ & $203(88.5)$ & \\
\hline Yes & $0(0.0)$ & $25(11.5)$ & \\
\hline $\begin{array}{l}\text { Family history } \\
\text { of CRC, no. (\%) }\end{array}$ & & & 0.54 \\
\hline Present & $2(9.5)$ & $38(17.6)$ & \\
\hline Absent & $19(90.5)$ & $190(82.4)$ & \\
\hline
\end{tabular}

${ }^{\text {a}}$ First-degree relatives. SPS, serrated polyposis syndrome; BMI, body mass index; ADA, advanced adenoma; CRC, colorectal cancer.

were 11 smokers in the SPS group (52.4\%). Using univariate logistic regression analysis, we analyzed the association between SPS and 7 independent variables, namely age, gender, BMI, alcohol consumption, smoking, family history of CRC and advanced adenoma [adenoma $\geq 1 \mathrm{~cm}$; villous component (tubulovillous or villous); and high-grade dysplasia]/CRC.

Advanced adenoma/CRC. Synchronous advanced adenoma/CRC was observed in 76 of the 249 patients (30.5\%). The OR for synchronous advanced adenoma/CRC was higher in patients with compared to those without SPS, but there was 
no significant association between synchronous advanced adenoma/CRC and SPS.

\section{Discussion}

The prevalence of SPS among patients with HPs in this study was $8.4 \%$, which was higher compared to that previously observed. This may be attributed to our use of chromoendoscopy, the most accurate method to date for determining the number of HPs. HPs are difficult to detect with conventional imaging techniques, as their color and appearance are similar to those of normal mucosa, unlike typical adenomatous polyps, and their vascular network is weak, unlike that of hypervascular adenomas. In addition, serrated polyps, mainly sessile serrated adenomas, are typically sessile or flat, making their detection even more difficult (17). Since these lesions have been shown to have malignant potential, particularly in the context of SPS, early endoscopic detection is crucial. In this regard, novel, more advanced endoscopic techniques, such as chromoendoscopy, become important. Chromoendoscopy in SPS cases should be performed by spraying a contrast dye over the entire surface of the colon and using a magnification endoscope. The most widely used contrast dye is indigo carmine, which accumulates in the pits and innominate grooves of the colonic mucosa, outlining the borders of flat lesions and forming the described Kudo patterns (18). Hyperplastic and serrated polyps typically exhibit Kudo type II patterns.

Published randomized trials have shown that high-definition pancolonic chromoendoscopy almost doubles the rate of detection of sporadic serrated polyps compared to conventional endoscopy (18-23). In these studies, HPs were found in $20 \%$ of patients undergoing chromoendoscopy vs. $10 \%$ of those undergoing conventional endoscopy and this difference was statistically significant. In addition, our study was performed on patients visiting an independent cancer center, who would be expected to be at greater risk of CRC compared to the general public.

Two types of diagnostic criteria were used for the endoscopic detection of SPS: i) $\geq 5$ HPs, with at least 2 polyps $>10 \mathrm{~mm}$ in diameter; and ii) the presence of numerous HPs, regardless of size. In patients meeting the former criterion, the HPs are mainly located in the right colon, whereas, in those meeting the latter, the majority of HPs are located in the left colon. All the patients with SPS fulfilled the latter criterion and none fulfilled the former, suggesting that the diagnostic criteria for SPS require revision.

In assessing the developmental path of CRC, we found that the former criterion for SPS ( $\geq 5$ HPs, with at least 2 polyps $>10 \mathrm{~mm}$ in diameter) corresponded to the serrated pathway. By contrast, it has not yet been determined whether $\mathrm{CRC}$ in patients meeting the latter criterion (numerous HPs, regardless of size) develops through the serrated pathway. A number of our SPS patients had local lesions, either advanced adenoma or CRC, in the left colon. We also observed that the incidence of advanced adenoma/CRC was significantly higher in patients with multiple small HPs compared to that in patients without HPs, indicating that SPS may be a risk factor for CRC.

Our results suggested that the risk factors for SPS include older age and higher BMI, but not gender, alcohol and smoking. In another study, however, cigarette smoking was found to be strongly associated with SPS, suggesting that smoking may increase the occurrence of this condition (24). Although an epidemiological study reported associations between CRC and alcohol consumption and smoking (25), these findings were not confirmed by our study. Not all CRCs arise from SPS; thus, SPS should be considered as a risk factor for CRC, even in patients who do not smoke or drink.

We observed that the percentage of patients with a family history of CRC was higher in the SPS group compared to that in the non-SPS group, although the difference was not statistically significant. Giant HP has been reported as a new familial syndrome predisposing to colorectal cancer (26). SPS criteria may be useful for identifying patients who should undergo screening colonoscopy.

Although the difference was not statistically significant, the rate of advanced adenoma/CRC was higher in the SPS compared to that in the non-SPS group. The rate of CRC in the entire cohort was higher compared to that previously reported, with some of these patients having SPS. Thus, patients with SPS should be prospectively screened for CRC.

There were several limitations to this study. First, all the patients were evaluated at a single institution, namely the National Cancer Center, Tokyo, Japan, which may have introduced a selection bias. Second, the sample size was relatively small, particularly for patients with SPS. Third, the clinical characteristics of SPS patients were investigated in an exploratory manner and, therefore, cannot be considered to be the absolute clinical characteristics of SPS. The strength of this study, however, lies with its ability to identify HPs with high precision.

In conclusion, our results suggest that the risk factors for SPS include older age and higher BMI. The prevalence of advanced adenoma/CRC was higher among patients with SPS compared to those without SPS, although the difference was not statistically significant.

\section{Acknowledgements}

The authors would like to thank Editage for editing the manuscript.

\section{References}

1. Goldman H, Ming S and Hickock DF: Nature and significance of hyperplastic polyps of the human colon. Arch Pathol 89: 349-354, 1970

2. Young J, Jenkins M, Parry S, et al: Serrated pathway colorectal cancer in the population: genetic consideration. Gut 56: 1453-1459, 2007.

3. Sandmeier D, Benhattar J, Martin P and Bouzourene H: Serrated polyps of the large intestine: a molecular study comparing sessile serrated adenomas and hyperplastic polyps. Histopathology 55: 206-213, 2009.

4. Noffsinger AE: Serrated polyps and colorectal cancer: new pathway to malignancy. Annu Rev Pathol 4: 343-364, 2009.

5. Burt RW and Jass J: Hyperplastic polyposis. In: World Health Organization Classification of Tumours. Pathology and Genetics. Tumours of the Digestive System. Hamilton SR and Aaltonen LA (eds). Springer-Verlag, Berlin, pp135-136, 2000.

6. Chow E, Lipton L, Lynch E, et al: Hyperplastic polyposis syndrome: phenotypic presentations and the role of MBD4 and MYH. Gastroenterology 131: 30-39, 2006.

7. Warner AS, Glick ME and Fogt F: Multiple large hyperplastic polyps of the colon coincident with adenocarcinoma. Am J Gastroenterol 89: 123-125, 1994. 
8. Tonooka T, Sano Y, Fujii T, et al: Adenocarcinoma in solitary large hyperplastic polyp diagnosed by magnifying colonoscope: report of a case. Dis Colon Rectum 45: 1407-1411, 2002.

9. Yano T, Sano Y, Iwasaki J, et al: Distribution and prevalence of colorectal hyperplastic polyps using magnifying pan-mucosal chromoendoscopy and its relationship with synchronous colorectal cancer: prospective study. J Gastroenterol Hepatol 20: 1572-1577, 2005

10. Jorgensen H, Mogensen AM and Svendsen LB: Hyperplastic polyposis of the large bowel. Three cases and a review of the literature. Scand J Gastroenterol 31: 825-830, 1996.

11. Hyman NH, Anderson P and Blasyk H: Hyperplastic polyposis and the risk of colorectal cancer. Dis Colon Rectum 47: 2101-2104, 2004

12. Young JP and Parry S: Risk factors: Hyperplastic polyposis syndrome and risk of colorectal cancer. Nat Rev Gastroentero Hepatol 7: 594-595, 2010.

13. Kalady MF, Jarrar A, Leach B, et al: Defining phenotypes and cancer risk in hyperplastic polyposis syndrome. Dis Colon Rectum 54: 164-170, 2011.

14. Rashid A, Houlihan PS, Booker S, Petersen GM, Giardiello FM and Hamilton SR: Phenotypic and molecular characteristics of hyperplastic polyposis. Gastroenterology 119: 323-332, 2000.

15. Leggett BA, Devereaux B, Biden K, Searle J, Young J and Jass J: Hyperplastic polyposis: association with colorectal cancer. Am J Surg Pathol 25: 177-184, 2001.

16. Rubio CA, Stemme S, Jaramillo E and Lindblom A: Hyperplastic polyposis coli syndrome and colorectal carcinoma. Endoscopy 38 : 266-270, 2006

17. Wallace K, Grau MV, Ahnen D, et al: The association of lifestyle and dietary factors with the risk for serrated polyps of the colorectum. Cancer Epidemiol Biomarkers Prev 18: 2310-2317, 2009.

18. Kudo S, Tamura S, Nakajima T, Yamano H, Kusaka H and Watanabe H: Diagnosis of colorectal tumorous lesions by magnifying endoscopy. Gastrointest Endosc 44: 8-14, 1996.
19. Kahi CJ, Anderson JC, Waxman I, et al: High-definition chromocolonoscopy vs. high-definition white light colonoscopy for average-risk colorectal cancer screening. Am J Gastroenterol 105: 1301-1307, 2010

20. Brooker JC, Saunders BP, Shah SG, et al: Total colonic dye-spray increases the detection of diminutive adenomas during routine colonoscopy: a randomized controlled trial. Gastrointest Endosc 56: 333-338, 2002.

21. Hurlstone DP, Cross SS, Slater R, Sanders DS and Brown S: Detecting diminutive colorectal lesions at colonoscopy: a randomised controlled trial of pan-colonic versus targeted chromoscopy. Gut 53: 376-380, 2004.

22. Lapalus MG, Helbert T, Napoleon B, et al: Does chromoendoscopy with structure enhancement improve the colonoscopic adenoma detection rate? Endoscopy 38: 444-448, 2006.

23. Le Rhun M, Coron E, Parlier D, et al: High resolution colonoscopy with chromoscopy versus standard colonoscopy for the detection of colonic neoplasia: a randomized study. Clin Gastroenterol Hepatol 4: 349-354, 2006.

24. Walker RG, Landmann JK, Hewett DG, et al: Hyperplastic polyposis syndrome is associated with cigarette smoking, which may be a modifiable risk factor. Am J Gastroenterol 105: 1642-1647, 2010

25. Otani T, Iwasaki M, Yamamoto S, et al; Japan Public Health Center-based Prospective Study Group: Alcohol consumption, smoking, and subsequent risk of colorectal cancer in middle-aged and elderly Japanese men and women: Japan Public Health Center-based prospective study. Cancer Epidemiol Biomarkers Prev 12: 1492-1500, 2003

26. Jeevaratnam P, Cottier DS, Browett PJ, Van De Water NS, Pokos V and Jass JR: Familial giant hyperplastic polyposis predisposing to colorectal cancer: a new hereditary bowel cancer syndrome. J Pathol 179: 20-25, 1996. 